\title{
Numerical Study of Devolatilization Models on the Combustion Process of Pulverized Fuel Flow Swirling
}

\author{
Victor A. Kuznetsov $v^{\mathrm{a}}$, Alexander A. Dekterev ${ }^{\mathrm{a}, \mathrm{b}}$, \\ Andrey V. Sentyabov ${ }^{\mathrm{a}, \mathrm{b}}$ and Mikhail Y. Chernetskiy \\ ${ }^{a}$ Siberian Federal University \\ 79 Svobodny, Krasnoyarsk, 660041, Russia \\ ${ }^{b}$ Kutateladze Institute of Thermophysics \\ Novosibirsk, 630090, Russia
}

Received 02.06.2015, received in revised form 23.12.2015, accepted 02.06.2016

The results of computational studies of the effect devolatilization models in the processes of ignition and combustion of pulverized coal in the swirling flow at the shooting bench with capacity 2.4 MW. Consider a few devolatilization models in a one and a two-stage approach, as well as models based on the structural features of the structure of the coal substance. For the numerical simulation of turbulent flow of an incompressible fluid used Reynolds equation based on the interfacial interactions. To close the Reynolds equations used a standard two-parameter model of turbulence $k-\varepsilon$. To solve the equation of heat radiation used P1 approximation of the method of spherical harmonics. To describe the optical properties of the gas model was used the sum of gray gases. To describe the processes of the movement of coal particles used the method of Lagrange. Burning char based on diffusion - kinetic approach. Comparative analysis of the results of mathematical modeling with experimental data showed that when choosing the correct values of the effective kinetic constants and parameters characterizing the structure of the carbon material, a model release volatiles are consistent with each other and with the experimental data on the distribution of temperature and composition of gases in the combustion chamber.

Keywords: mathematical modeling, pulverized fuel, swirling flow, fire stand, devolatilization model.

Citation: Kuznetsov V.A., Dekterev A.A., Sentyabov A.V., Chernetskiy M.Y. Numerical Study of Devolatilization Models on the Combustion Process of Pulverized Fuel Flow Swirling, J. Sib. Fed. Univ. Eng. technol., 2016, 9(1), 15-23. DOI: 10.17516/1999-494X-2016-9-1-15-23.

(C) Siberian Federal University. All rights reserved

* Corresponding author E-mail address: micch@yandex.ru 


\title{
Расчетное исследование влияния моделей
}

\section{выхода летучих веществ на процессы горения \\ пылеугольного топлива при закрутке потока}

\author{
В.А. Кузнецов ${ }^{\mathrm{a}}$ А.А. Дектерев ${ }^{\mathrm{a}, \boldsymbol{\sigma}}$, \\ А.В. Сентябов ${ }^{\mathrm{a}, \boldsymbol{\sigma}}$, М.Ю. Чернецкий ${ }^{\mathrm{a}, \boldsymbol{\sigma}}$ \\ ${ }^{a}$ Сибирский федеральный университет \\ Россия, 660041, Красноярск, пр. Свободный, 79 \\ ${ }^{6}$ Институт теплофизики им. С.С. Кутателадзе СО РАН \\ Россия, 630090, Новосибирск
}

Приведены результаты расчётных исследований влияния моделей выхода летучих веществ на процессы воспламенения и горения пылеугольного топлива при закрутке потока на огневом стенде мощностью 2.4 МВт. Рассмотрено несколько моделей выхода летучих веществ: в одностадийном и двухстадийном приближении, а также модели, основанной на структурных особенностях строения угольного вещества. Для численного моделирования турбулентного течения несжимаемой жидкости использовались уравнения Рейнольдса с учетом межфазного взаимодействия. Для замыкания уравнения Рейнольдса применена двухпараметрическая

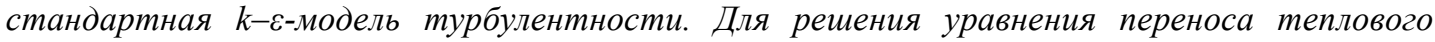
излучения взято P1-приближение метода сферических гармоник. Для описания оптических свойств газов была использована модель суммы серых газов. Для описания прочессов движения угольных частии обратились к методу Лагранжа.

Сравнительный анализ результатов математического моделирования с экспериментальными данными показал, что при выборе правильных значений эффективных кинетических констант, а также параметров, характеризующих структуру угольного вещества, модели выхода летучих веществ согласуются между собой и с экспериментальными данными по распределению температур и составу газов в топочной камере.

Ключевые слова: математическое моделирование, пылеугольное топливо, закрученное течение, огневой стенд, модель выхода летучих веществ.

\section{Введение}

Математическое моделирование топочных устройств является на текущий момент одним из важнейших способов получения наиболее представительной информации об аэродинамике, локальном и суммарном теплообмене. Несмотря на значительные успехи, достигнутые в развитии численного эксперимента, большое разнообразие, до конца не изученная структура угля и сложные химические процессы, происходящие при горении и газификации угольного топлива, не позволяют создать универсальных моделей. Одним из процессов, определяющих процесс горения угольного вещества, является выход летучих веществ. Существует несколько модельных описаний данного процесса, построенных в основном на эмпирических, полуэмпирических зависимостях. Можно выделить несколько основных моделей процесса выхода летучих веществ, которые широко используются в составе комплексной математической модели процессов гидродинамики, тепломассообмена и кинетики термохимических превращений твердого органического топлива. Первая из них основана на законе Аррениуса с эффективными кинетическими константами. Данная модель определяет скорость по- 
тери массы с изменением температуры. Такие эмпирические модели широко используются в составе комплексных моделей [1] и требуют незначительных вычислительных ресурсов. Однако эмпирические основы данных моделей зачастую не позволяют их применять в тех температурных интервалах и интервалах скорости нагрева, для которых не были определены эффективные кинетические константы. С другой стороны, если можно экспериментально получить эффективные кинетические константы для интересующих условий, то данный подход дает результаты, сопоставимые с более сложными моделями как для пылеугольного потока [2], так и для отдельной частицы [3]. В настоящее время активно развивается использование в составе комплексных математических моделей моделей, описывающих процесс разложения органического вещества с учетом структуры угля и ее разрушения в процессе нагрева. Это такие модели, как Functional Group-Depolymerisation Vaporisation Cross-linking (FG-DVC) и Chemical Percolation Devolatilisation (CPD) [4-6]. Основой данных моделей является представление органической массы угля в виде фрагментов разной степени ароматизированности, соединённых «мостиками», которые представляют собой алифатические цепи, эфирные, тиоэфирные или их комбинации. Отличительными особенностями двух моделей служит то, что модель FG-DVC позволяет представить летучие компоненты в виде смеси газов, тогда как в модели нет разделения на многокомпонентный газ. Исходные характеристики угля для модели CPD рассчитываются по корреляциям. Для обеспечения более точного исходного описания строения угля можно использовать экспериментальные данные ЯМРспектроскопии.

В настоящей статье представлен сравнительный анализ влияния нескольких наиболее популярных моделей выхода летучих веществ на процессы воспламенения и горения пылеугольного топлива при закрутке потока.

\section{Постановка задачи \\ и математическая модель}

Для верификации математической модели использованы экспериментальные данные по горению пылеугольного факела при наличии закрутки потока на огневом стенде мощностью 2.4 МВт [7]. Схема огневого стенда представлена на рис. 1. Расходные характеристики при проведении экспериментальных исследований и использованные в расчетах составляли: первичный воздух (сухой) 0.117 кг/с, температура 343.15 К, средняя аксиальная скорость 23.02 м/c, расход угля (сухого) 0.073 кг/с. Расход вторичного воздуха (сухого) 0.745 кг/с, температура $573.15 \mathrm{~K}$, средняя аксиальная скорость 43.83 м/с, средняя тангенциальная скорость 49.42 м/с. Технический состав угля (мас. \%, сухой): летучие вещества 37.4, связанный углерод (fixed carbon) $54.3, \mathrm{~A}^{\mathrm{c}}-8.3$. Химический состав угля (мас. \%, рабочая): $\mathrm{C}^{\mathrm{r}}-80.36, \mathrm{H}^{\mathrm{r}}-5.08, \mathrm{~N}^{\mathrm{r}}-1.45$, $\mathrm{S}^{\mathrm{r}}-0.94, \mathrm{O}^{\mathrm{r}}-12.17 . \mathrm{Q}_{\mathrm{H}}=32.32 \mathrm{MДж/кг.}$

Для численного моделирования турбулентного течения несжимаемой жидкости использовали уравнения Рейнольдса с учетом межфазного взаимодействия. Ранее проведенные исследования показали, что двухпараметрические модели турбулентности: стандартная $k-\varepsilon$-модель турбулентности, $k-\omega$ SST модели Ментера, а также модель напряжения Рейнольдса дают схожие результаты для рассматриваемой задачи. 


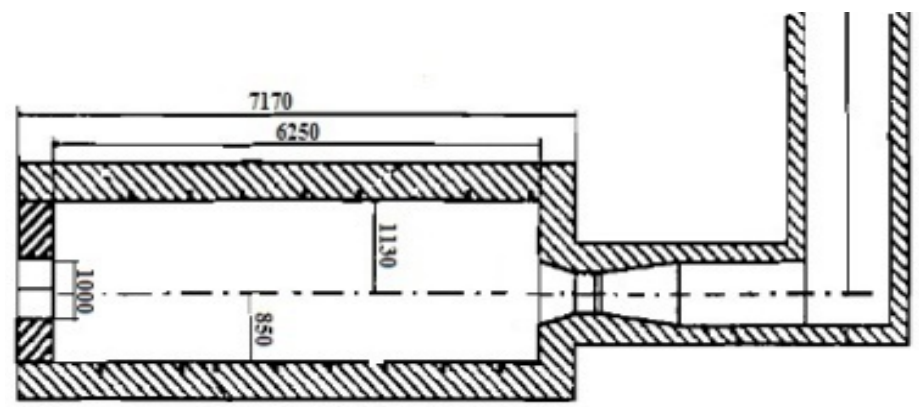

Рис. 1. Огневой стенд [7]

В настоящей статье для описания процессов движения частицы взят метод Лагранжа. Движение частицы описывается уравнениями динамики материальной точки с учетом силы сопротивления и силы тяжести. Учет турбулентности потока при движении частицы производится введением случайных флуктуаций скорости газа в уравнение движения для частиц. Решение уравнения переноса лучистой энергии базируется на P1 аппроксимации метода сферических гармоник для серой двухфазной двухтемпературной среды. Как показали ранее выполненные расчетные исследования [8-10], данная комплексная модель дает хорошее согласование при сжигании твердого органического топлива в пылевидном состоянии.

Процесс горения угольной частицы рассматривается в виде последовательных этапов: испарение влаги из топлива, выход и горение летучих компонент и горение коксового остатка. Испарение влаги из топлива рассчитывается на основе значения количества водяного пара на поверхности частицы и в объеме.

Выбор модели выхода летучих веществ играет основную роль для правильной оценки процесса воспламенения угольной частицы. Существует достаточно много подходов к описанию этого процесса. В данной статье рассмотрены наиболее широко используемые модели выхода летучих веществ. Это одностадийное и двухстадийное приближение процесса, а также модель, основанная на структурной особенности строения угольного вещества.

При одностадийном приближении скорость выхода летучих веществ определятся по закону Аррениуса с эффективными кинетическими константами:

$$
k_{v}=k_{v, 0} e^{-\left(E_{v} / R T_{p}\right)},
$$

где $k_{v, 0}$ - предэкспоненциальный множитель процесса выхода летучих веществ; $E_{v}-$ энергия активации процесса выхода летучих веществ. Значения констант взяты из работы [7] $k_{v, 0}=1.3 \cdot 10^{7}$ $(1 / \mathrm{c}), E_{v}=1.67 \cdot 10^{8}$ (Дж/Кмоль).

При рассмотрении двухстадийного механизма, на основе модели Кобайаши [11], предполагается наличие двух разных по степени связанности с органической массой компонент летучих веществ, характеризующихся разными кинетическими параметрами:

$$
\frac{m_{v}(t)}{\left(1-f_{w, 0}\right) m_{p, 0}-m_{a}}=\int_{0}^{t}\left(\alpha_{1} k_{1}+\alpha_{2} k_{2}\right) \exp \left(-\int_{0}^{t}\left(k_{1}+k_{2}\right) d t\right) d t .
$$




\section{Рассматривались следующие наборы констант:}

Kobayashi et al. [11]: $\alpha_{1}-0.3, \mathrm{~A}_{1}-2 \cdot 10^{5}(1 / \mathrm{c}), \mathrm{E}_{1}-1.05 \cdot 10^{8}$ (Дж/Кмоль), $\alpha_{2}-1, \mathrm{~A}_{2}-1.3 \cdot 10^{7}(1 / \mathrm{c})$, $\mathrm{E}_{2}-1.67 \cdot 10^{8}$ (Дж/Кмоль).

Cho et al. [12]: $\alpha_{1}-0.38, \mathrm{~A}_{1}-3.7 \cdot 10^{5}(1 / \mathrm{c}), \mathrm{E}_{1}-7.4 \cdot 10^{7}$ (Дж/Кмоль), $\alpha_{2}-0.8, \mathrm{~A}_{2}-1.46 \cdot 10^{13}(1 / \mathrm{c})$, $\mathrm{E}_{2}-2.5 \cdot 10^{8}$ (Дж/Кмоль).

Ubhayakar et al. [13]: $\alpha_{1}-0.292, \mathrm{~A}_{1}-3.7 \cdot 10^{5}$ (1/c), $\mathrm{E}_{1}-7.4 \cdot 10^{7}$ (Дж/Кмоль), $\alpha_{2}-0.438, \mathrm{~A}_{2}-$ $1.46 \cdot 10^{13}(1 / \mathrm{c}), \mathrm{E}_{2}-2.5 \cdot 10^{8}$ (Дж/Кмоль), где $\alpha_{1}$ и $\alpha_{2}-$ коэффициенты, определяющие долю «низкотемпературных» и «высокотемпературных» летучих веществ.

При рассмотрении модели с учетом структуры угольного вещества органическая масса угля представляется в виде фрагментов разной степени ароматизированности, соединённых «мостиками», которые представляют собой алифатические цепи, эфирные, тиоэфирные или их комбинации. В работе используется CPD (chemical percolation devolatilization) модель [14]. В данной модели в так называемой подвижной фазе под действием температуры происходит разрушение «мостиков», часть фрагментов отщепляется от ядерной части и в виде низкомолекулярных паро- и газообразных соединений переходит в летучие вещества. Часть фрагментов, имеющая более тяжелый молекулярный вес, переходит в плавкое состояние (смолистые вещества), рекомбинирует, частично образуются летучие вещества, а также образуют с ядерной частью коксовый остаток. Параметры, используемые для данной модели, были взяты на основе корреляций, предложенных в работе [15]. На основе данных корреляций в модели в качестве исходных параметров задавалось общее количество «мостиков» в структуре угля в виде их доли; количество «мостиков»- углеродных связей, которые возникают при рекомбинации смолистых веществ с ядерной частью, - координационное число, определяющее структуру угля, а также масса кластера и масса боковых цепей.

\section{Результаты исследования влияния модели выхода летучих веществ}

На основе полученных выше результатов для дальнейших расчетов была выбрана двухпараметрическая модель турбулентности $k-\varepsilon$. Результаты расчета с использованием разных моделей выхода летучих веществ представлены на рис. 2-4. Можно видеть, что в общем случае одностадийный, двухстадийный механизмы описания выхода летучих веществ, а также многостадийная модель CPD дают схожие результаты. Это объясняется прежде всего характером течения вблизи горелочного устройства, где существует обратное течение горячих газов, которое и является основным инициатором воспламенения угольной пыли. Но имеются и отличия в результатах расчетов. Так, двухстадийная модель с кинетическими константами, предлагаемая в работе [11], дала более плохое соответствие с экспериментальными данными как по распределению температур (рис. 2), так и по концентрации кислорода (рис. 3) и углекислого газа (рис. 4). При этом результаты расчетов по двухстадийной модели с кинетическими константами из работы [13] хорошо согласуются с экспериментальными данными. В целом результаты расчётов с разными моделями выхода летучих похожи, за исключением двухстадийной модели с кинетическими константами [11].

Скорость выхода летучих веществ с использованием параметров [11] для двухстадийной модели значительно отличается от других моделей, что связано в первую очередь с распределением между низкотемпературным выходом летучих веществ и термической деструкцией 
угольного вещества, имеющей место при более высоких температурах. На рис. 5 видно, что если для всех моделей выход летучих локализован на начальном участке факела, то для двухстадийной модели выхода летучих веществ [11] имеется две ярко выраженные области выхода летучих веществ - на начальном участке факела и дальше по потоку. При этом общее количество летучих веществ, образующихся из угля по модели [11], больше, чем для остальных моделей, что определяет меньшую концентрацию кислорода и температуры по длине камеры сгорания (рис. 2, 3).

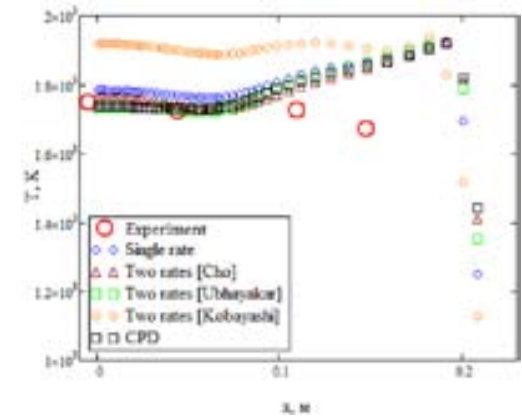

a) $z=0$

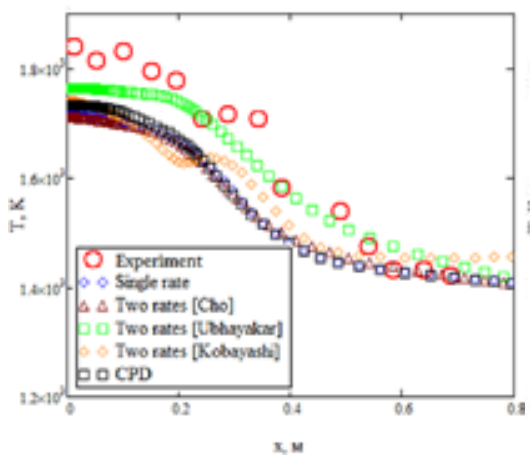

в) $\mathrm{z}=0.85$

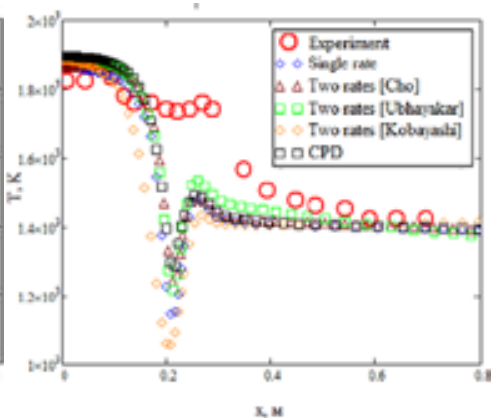

б) $\mathrm{z}=0.25$

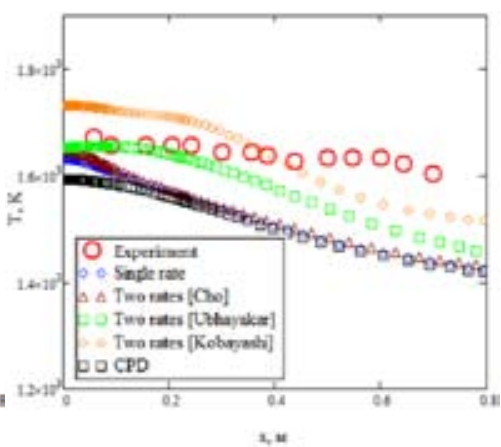

г) $\mathrm{z}=1.95$

Рис. 2. Распределение температуры по длине топочной камеры в разных сечениях: $\mathrm{z}$ - расстояние от горелки

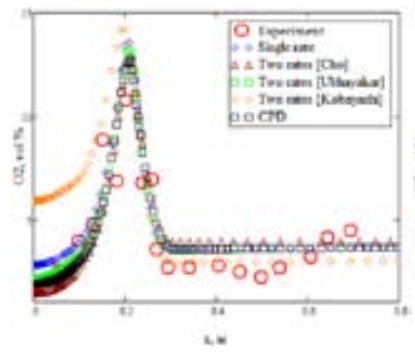

a) $\mathrm{z}=0.25$

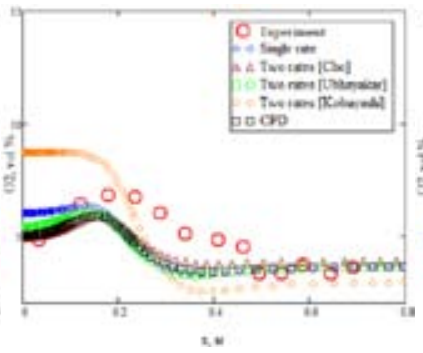

б) $\mathrm{z}=0.85$

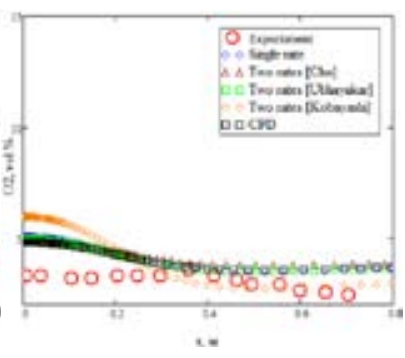

в) $\mathrm{z}=1.95$

Рис. 3. Распределение концентрации кислорода по длине топочной камеры в разных сечениях: $\mathrm{z}-$ расстояние от горелки 


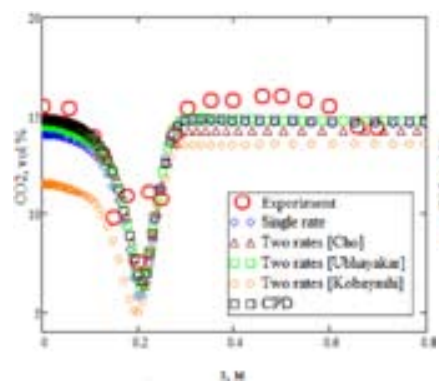

a) $\mathrm{z}=0.25$

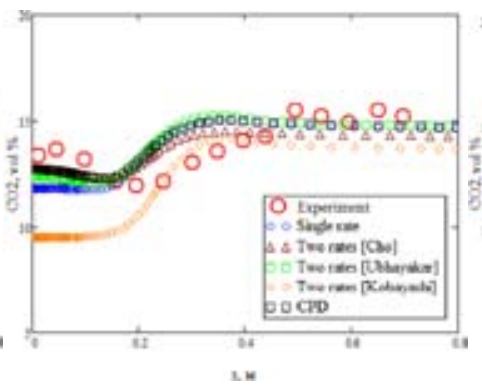

б) $\mathrm{z}=0.85$

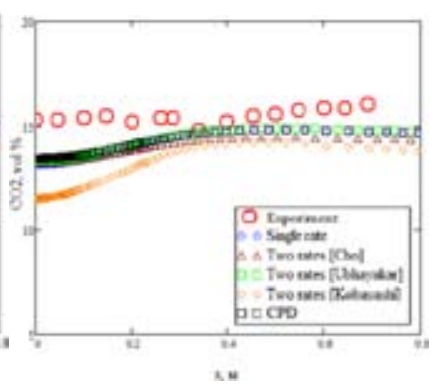

в) $\mathrm{z}=1.95$

Рис. 4. Распределение концентрации углекислого газа по длине топочной камеры в разных сечениях: $\mathrm{z}$ расстояние от горелки

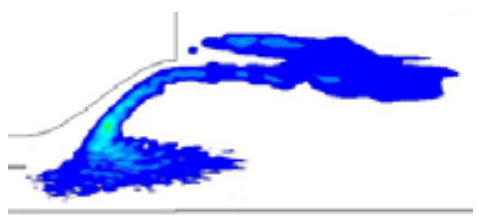

а) одностадийное приближение выхода летучих веществ

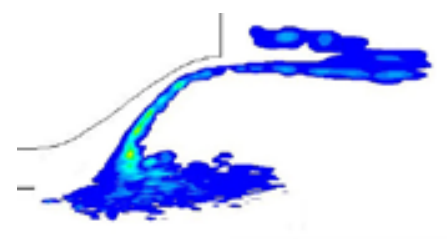

в) двухстадийная модель выхода летучих веществ[12]

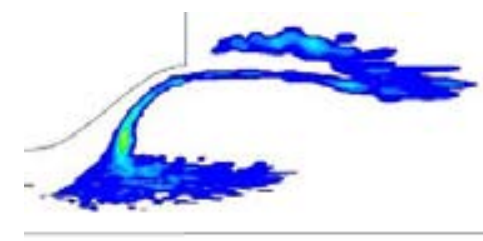

б) двухстадийная модель выхода летучих веществ[13]

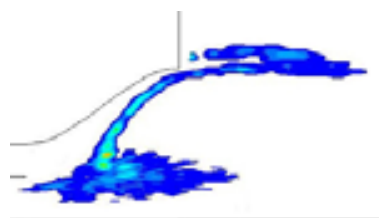

г) многостадийная модель CPD [14]

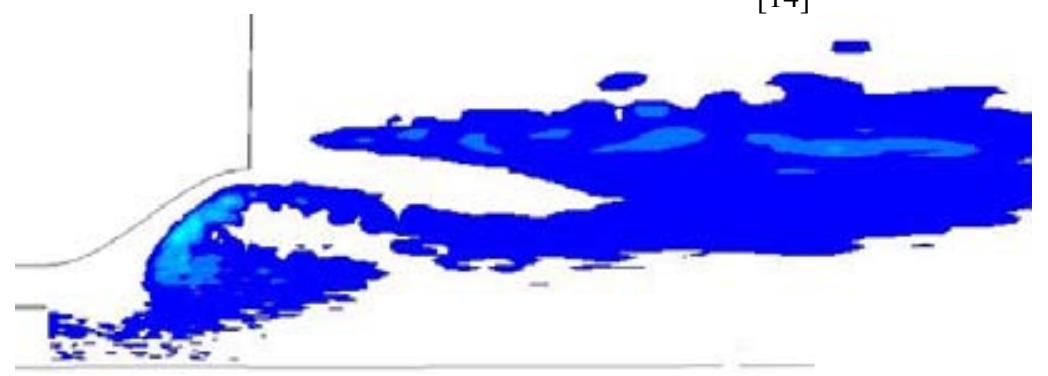

д) двухстадийная модель выхода летучих веществ [11]

Рис. 5. Поле скорости выхода летучих веществ в центральном сечении топочной камеры при разных моделях выхода летучих веществ, кг/с

\section{Выводы}

Сравнительный анализ влияния моделей выхода летучих веществ на процессы воспламенения и горения пылеугольного топлива в топочной камере с вихревой горелкой показал, что выбор данных моделей может оказать существенное влияние на результаты расчетов. Несмо- 
тря на то что закрутка потока создает зону обратного тока в центре факела, в результате чего происходит подсос горячих горючих газов и создается устойчивое воспламенение угольной пыли, ряд моделей, используемых в литературе, не позволяет достоверно описать процесс выхода летучих веществ в данных условиях. Это связано в первую очередь с выбором эффективных кинетических констант для данного процесса. Поэтому при наличии эффективных кинетических констант, определяющих процесс выхода летучих веществ для рассматриваемого угольного топлива, достаточно использовать модели, основанные как на одностадийном, так и на двухстадийном приближении. В противном случае необходимо использовать модели, описывающие процесс выхода летучих веществ с учетом структуры угля и ее разрушения в процессе нагрева, в том числе с применением представленных в литературе корреляций.

\section{Исследования выполнены при поддержке РФФИ (грант № 15-38-20969).}

\section{Список литературы}

[1] Eaton A.M., Smoot L.D., Hill S.C., Eatough C.N. Components, formulations, solutions, evaluation, and application of comprehensive combustion models, Prog. Energy Combust. Sci., 1999, $25,387-436$.

[2] Hart J., Al-Abbas A.H., Naser J. Numerical investigation of pyrolysis of a LoyYang coal in a lab-scale furnace at elevated pressures, Heat Mass Transfer, 2013, 49, 1725-1732.

[3] Wendt C., Eigenbrod C., Moriue O., Rath H.J. A model for devolatilization andignition of an axisymmetric coal particle, Proc. Combust. Inst. 2002, 29, 449-457.

[4] Williams A, Backreedy R, Habib R, Jones J.M., Pourkashanian M. Modelling coal combustion: the current position, Fuel, 2002, 81, 605-618.

[5] Backreedy R.I., Fletcher L.M., Pourkashanian M., Williams A. Modeling pulverized coal combustion using a detailed coal combustion model, Combust Sci. Technol., 2006, 178, 763-787.

[6] Chern J.S., Hayhurst A.N. A simple theoretical analysis of the pyrolysis of an isothermal particle of coal, Combust Flame, 2010, 157, 925-933.

[7] Peters A.A.F., Weber R. Mathematical Modeling of a 2.4 MW Swirling Pulverized Coal Flame, Combustion Science and Technology, 1997, 122. I(1-6), 131-182.

[8] Чернецкая Н.С.; Чернецкий М.Ю.; Дектерев А.А. Численное исследование влияния термоподготовки угля на образование оксида азота в процессе горения. Журнал СФУ. Техника и технологии, 2014, 7(1), 83-95 [Chernetskaya N.S., Chernetskiy M. Yu., Dekterev A.A. Numerical Investigation of Influence Thermal Preparation Coal on Nitric Oxides Formation in Combustion Process, J. Sib. Fed. Univ. Eng. technol., 2014, 7(1), 83-95 (in Russian)]

[9] Чернецкий М.Ю., Дектерев А.А., Бурдуков А.П. Расчетное исследование факельного горения мелкодисперсного лигноцеллюлозного сырья. Журнал СФУ. Техника и технологии, 2013, 6(6), 625-636 [Chernetskiy M. Yu., Dekterev A.A., Burdukov A.P. Numerical Research of Pulverized Combustion of Micro-Grinded Lignocellulose Raw Materials, J. Sib. Fed. Univ. Eng. technol., 2013, 6(6), 625-636 (in Russian)]

[10] Chernetskiy, M.Yu., Dekterev, A.A., Burdukov A.P., Hanjalic K. Computational modeling of autothermal combustion of mechanically-activated micronized coal, Fuel, 2014, 135, 443-458.

[11] Kobayashi H., Howard J.B., Sarofim A.F., Proc. Combust. Inst. 1977, 16, 411-425. 
[12] Cho C.P., Jo S., Kim H.Y., Yoon S.S., Numer. Heat Transfer, Part A: Appl. 2007, 52, 11011122.

[13] Ubhayakar S.K., Stickler D.B., von Rosenberg C.W., Gannon R.E., Proc. Combust. Inst., 1976, 16, 427-436.

[14] Fletcher T.H., Kerstein A.R., Pugmire R.J., Grant D.M. Energy Fuels, 1990, 4, 54-60.

[15] Genetti D., Fletcher T.H. Predicting C NMR measurements of chemical structure based on the elemental composition and volatile matter content of coal. Utah: Brigham Young University, Department of Chemical Engineering, 1998. 